\title{
Is deep vein thrombosis different during pregnancy?
}

\author{
Risto Kaaja MD PhD \\ Previously published at www.cmaj.ca
}

$\infty \quad$ See related research article by Chan and colleagues, page 657

$\mathrm{T}$ he causes of deep vein thrombosis include damage of the vessel wall, stasis or low blood flow, and hypercoagulability, as alluded to in Virchow's triad. All of these factors can present during pregnancy. Hypercoagulability can be enhanced by the presence of hereditary thrombophilia, immobilization and operation (i.e., cesarean section). ${ }^{1}$ Could this cluster of risk factors lead to a different form of deep vein thrombosis in terms of the site of the thrombosis and propagation during pregnancy?

In a systematic review of studies covering a search period of 1966 to 2009, Chan and colleagues ${ }^{2}$ found that the distribution of deep vein thrombosis was different in pregnant women than in other patients. They confirmed earlier findings that deep vein thrombosis mainly affects the left leg and is usually isolated to the proximal iliac and/or femoral veins. ${ }^{3.4}$ Are these results real, and if so, how can they be explained?

First, we lack evidence-based studies on diagnostic techniques for detecting deep vein thrombosis in pregnant women. Because of the risk of fetal radiation and the concern about administering contrast agents, there are few studies of venography, the reference standard, being performed in pregnant women.

It was not surprising that Chan and colleagues could retrieve only 6 articles, from an initial list of 1098, on 124 pregnant women with deep vein thrombosis. Therefore, reporting and selection bias are possible. Deep vein thrombosis was diagnosed by venography in only 55 patients, compared with 69 patients in whom it was diagnosed with compression ultrasonography. It is possible that in the latter group, distal deep vein thromboses were missed.

Compared with venography, compression ultrasonography is highly sensitive $(97 \%)$ in detecting proximal vein thrombosis but less sensitive in detecting thrombosis limited to the calf. ${ }^{5}$ Magnetic resonance imaging, which is widely used for other indications, does not offer any better results. It has equivalent sensitivity and specificity to ultrasonography, but it has been evaluated in many fewer studies, using various techniques. ${ }^{6}$ The ideal study design with which to investigate the distribution of deep vein thrombosis in pregnant women and validate compression ultrasonography versus venography may be one in which the two procedures are systematically performed in pregnant women with acute deep vein thromboses. Such a study is not feasible.

\section{Key points}

- Evidence-based studies on diagnostic techniques for pregnant women in whom deep vein thrombosis is suspected are lacking.

- Isolated proximal deep vein thrombosis (without involvement in the calf veins) seems to be more common during pregnancy.

- The site of deep vein thrombosis and propagation can vary depending on individual risk factors for deep vein thrombosis (thrombophilic status) and fibrinolytic capacity.

Second, one of the main hypotheses of the study by Chan and colleagues was to determine whether the results of the prospective studies in nonpregnant women showing that most deep vein thromboses in the legs originate in the calf and progress proximally are valid for pregnant women. The results showed that isolated, proximal deep vein thrombosis (without involvement of the calf veins) seems to be more common during pregnancy. But even in nonpregnant women, proximal propagation of deep vein thrombosis from the calves into the popliteal or thigh veins can vary and can be less than $5 \% .^{7}$ The natural course of distal or proximal deep vein thrombosis in pregnancy is even less known.

Third, perhaps there is no common "natural course" of deep vein thrombosis developing in pregnancy. In each instance, the site of the thrombosis and propagation can vary depending on individual risk factors for the condition: thrombophilic status, possible immobilization and varying degrees of the effect of a growing uterus on venous stasis in the lower limbs. Also, the fibrinolytic capacity of the woman may play a role. ${ }^{8}$ In one recent study, isolated distal deep vein thrombosis was associated more often with transient risk factors (recent surgery, plaster immobilization and travel), whereas proximal deep vein thrombosis was associated more often with chronic conditions (active cancer, congestive heart failure and respiratory insufficiency in patients more than 75 years old). ${ }^{9}$ Pregnancy could contribute to a more chronic and

Risto Kaaja is from the Department of Medicine, Turku University, Satakunta Central Hospital Pori, Finland

CMAJ 2010. DOI:10.1503/cmaj.100279

All editorial matter in CMAJ represents the opinions of the authors and not necessarily those of the Canadian Medical Association. 
thrombophilic state and therefore predispose to proximal deep vein thromboses. Thrombophilia status, which was unknown in Chan and colleagues' study, could play a role in the initial site and propagation of deep vein thrombosis. ${ }^{10}$

The results of Chan and colleagues' study suggest that the distribution of deep vein thrombosis in pregnant women may differ from that in nonpregnant women. The increased prevalence of isolated proximal deep vein thrombosis is clinically important because of the high risk of pulmonary embolism (40\%-50\%). ${ }^{11}$ These findings should be confirmed by larger prospective studies.

Competing interests: None declared.

\section{REFERENCES}

1. Greer I. Inherited thrombophilia and venous thromboembolism. Best Pract Res Clin Obstet Gynaecol 2003; 17:413-25.

2. Chan WS, Spencer FA, Ginsberg JS. Anatomic distribution of deep vein thrombosis in pregnancy. CMAJ 2010;182:657-60.

3. Ray J, Chan WS. Deep vein thrombosis during pregnancy and the puerperium: a meta-analysis of the period of risk and the leg of presentation. Obstet Gynecol Surv 1999;54:265-71.

4. James AH, Tapson VF, Goldhaber SZ. Thrombosis during pregnancy and the postpartum period. Am J Obstet Gynecol 2005;193:216-9.

5. Kearon C, Julian JA, Ginsberg JS, et al. Noninvasive diagnosis of deep venous thrombosis. Ann Intern Med 1998;128:663-77.

6. Sampson FC, Goodacre SW, Thomas SM, et al. The accuracy of MRI in diagnosis of suspected deep vein thrombosis: systematic review and meta-analysis. Eur Radiol 2007; 1:175-81

7. Masuda EM, Kessler DM, Kistner RL, et al. The natural history of calf vein thrombosis: lysis of thrombi and development of reflux. J Vasc Surg 1998;28:67-73.

8. Hellgren M. Hemostasis during normal pregnancy and puerperium. Semin Thromb Hemost 2003;29:125-30.

9. Galanaud JP, Sevestre-Pietri MA, Bosson JL, et al. Comparative study on risk factors and early outcome of symptomatic distal versus proximal deep vein thrombosis: results from the OPTIMEV study. Thromb Haemost 2009;102:493-500.

10. Spiezia L, Tormene D, Pesavento R, et al. Thrombophilia as a predictor of persistent residual vein thrombosis. Haematologica 2008:93:479-80.

11. Girard P, Decousus M, Laporte S, et al. Diagnosis of pulmonary embolism in patients with proximal deep vein thrombosis: specificity of symptoms and perfusion defects at baseline and during anticoagulation therapy. Am J Respir Crit Care Med 2001;164:1033-7.

Correspondence to: Dr. Risto Kaaja, Professor of Medicine,

Turku University/ Satakunta Central Hospital, Sairaalantie 3, 28500 Pori, Finland; risto.kaaja@utu.fi
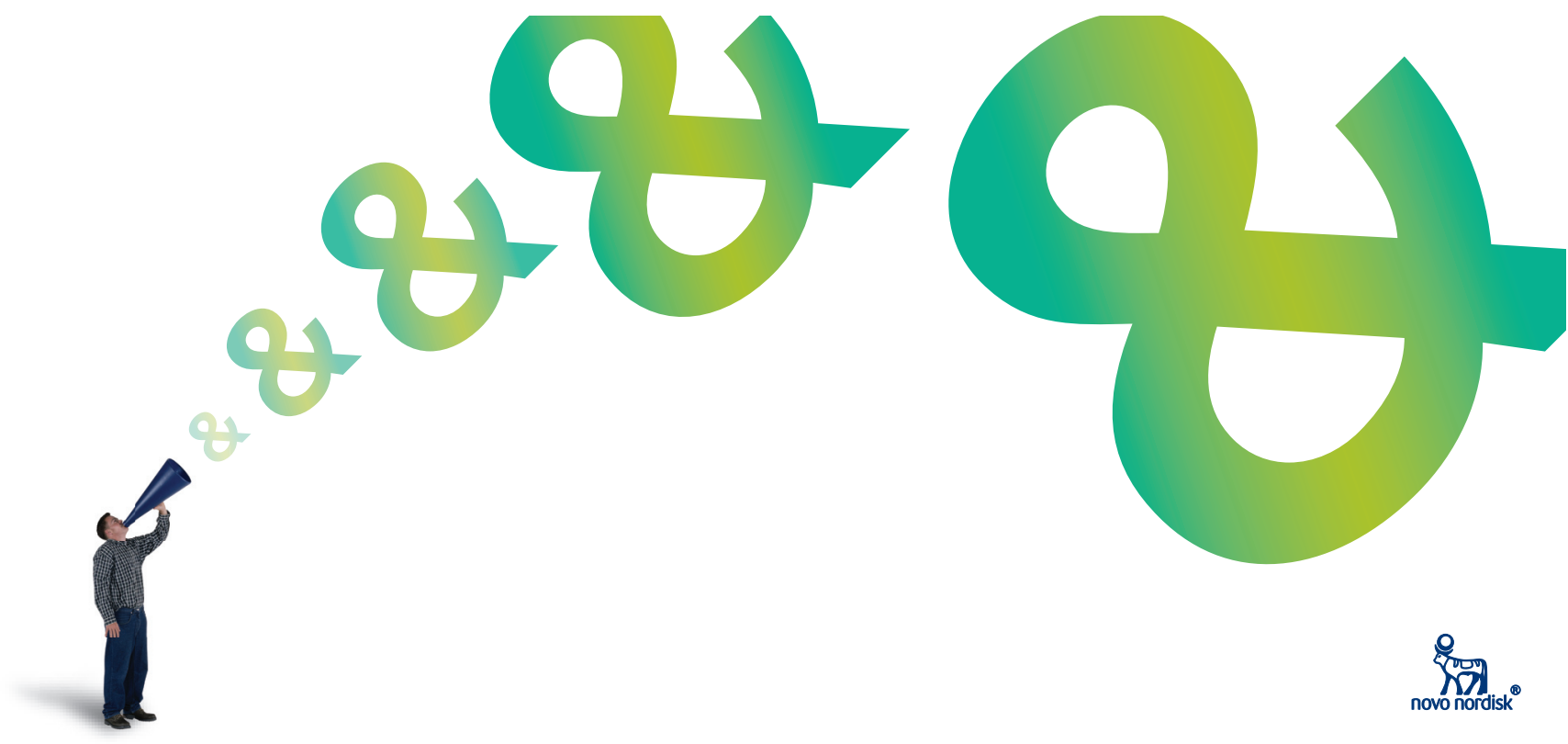